\title{
Barcode Localization using Curvelet Transform and Neural Network
}

\author{
Priyanka Gaur \\ M. Tech (CSE) \\ MITS, Laxmangarh
}

\author{
Shamik Tiwari \\ Assistant Professor \\ MITS, Laxmangarh
}

\begin{abstract}
Barcode localization is the main challenge in developing an imagebased barcode reading system. Bar codes are able to carry both explicit information and a database key, by encoding a series of characters or symbols. This paper deals with localization of European Article Number-13 (EAN-13) barcode in an image. A new approach for detecting and locating bar-codes is introduced here, which is based on the curvelet transform. All extracted feature by curvelet transform are applied to the neural network for training and testing. The performance of the proposed work shows efficient result.
\end{abstract}

Keywords: EAN-13 Barcode, Curvelet transform, Neural Network.

\section{INTRODUCTION:}

Barcodes are becoming more and more common and popular in just about every phase of consumer life. Every item on the market is labeled with barcode, which provide a tactics to track and store information of them. The success of barcode technology is due to its ability to encode information about product, manufacturer, country etc. in a compressed fashion. As we all see in supermarkets that information about a product such as price and description can be obtained by laser barcode scanner quickly. The one main deficiency of barcode scanners is lack of mobility. At the place of these laser scanners smart phones can be used, no doubt the use of smart phones is becoming increasingly widespread, and there features also are rapidly growing. For instance, they can facilitate users to access reviews or price comparisons for a product from a picture of its barcode while still in the store. So, it is necessary to first locate the appropriate position of the bar code, which is very complicating process since a typical image may contain other text and graphics.
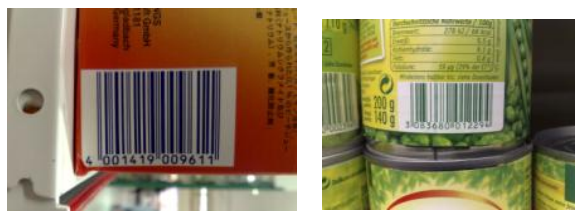

Fig1: Example of EAN-13 barcode images captured by smart phones.

Image processing techniques provide an efficient tool to localize barcode region from mobile captured images as shown in fig 1 . Rocholl, et al. [1] proposed a algorithm that locates the barcode in the camera image and extracts a scan line of brightness values. They find a straight line that intersects all bars. It does not have to be perpendicular to the bars, but it must go across the entire barcode present on the captured image. En Peng, et al. [2] uses a technique that detects the 1D barcode patterns which is formed by a rectangular array of parallel lines. Douglas Chai and Florian Hock [3] proposed a block-based technique for finding the barcode location. Within each block orientation of each labeled region is calculated and compared in order to test for parallel property.
Melinda Katona, et. al. [4] applied some morphological operation to localize and segment barcode region from an image. In this paper, we present curvelet transform technique for extracting features which are used to localize and segment barcode image at block level effectively.

\subsection{Curvelet Transform:}

In the field of image processing concept of curvelet transform is becoming popular. For multi-scale object representation Curvelet is a non-adaptive technique. To overcome the inherent limitations of wavelet like transforms, a geometric transform developed by Candes et al. [5], is known as Curvelet transform. With needle shaped basis functions curvelet transform is a multi-scale and multi-directional transform. In the wavelet transform Basis functions are isotropic, so it requires large number of coefficients to represent the curve singularities. In comparison to wavelet transform basis functions of curvelet transform are needle shaped and have high directional sensitivity and anisotropy. The main advantage of Curvelet Transform is, it allow representing edges and other singularities along lines in a more efficient way when compared with other transforms. And EAN-13 barcode is made up of black and white parallel edges, which can also be detected by curvelet statistical features.

With spatial variable (x) and a frequency domain variable (w) curvelet transform works in two dimensions. The polar coordinates in the frequency-domain are $\mathrm{r}$ and $\mathrm{t}$. A pair of windows: radial window $(\mathrm{W}(\mathrm{r}))$ and angular window $(\mathrm{V}(\mathrm{t}))$ is used to define curvelet transform [6].These windows will obey the admissibility conditions as illustrated in equations (1) and (2):

$$
\begin{aligned}
& \sum_{j=-\infty}^{\infty} W^{2}\left(2^{j} r\right)=1, r \in\left(\frac{3}{4}, \frac{3}{2}\right) \\
& \sum_{l=-\infty}^{\infty} V^{2}(t-l)=1, t \in\left(-\frac{1}{2}, \frac{1}{2}\right)
\end{aligned}
$$

$\mathrm{Uj}$ represents a polar wedge which is supported by the radial windows $\mathrm{W}$ and angular windows $\mathrm{V}$. $\mathrm{Uj}$ is defined in the Fourier domain by equation (3):

$\mathrm{U}_{\mathrm{j}}(\mathrm{r}, \theta)=2^{-(3 \mathrm{j} / 4)} \mathrm{W}\left(2^{-\mathrm{j}} \mathrm{r}\right) \mathrm{V}\left(\frac{2^{-\mathrm{j} / 2} \theta}{2 \Pi}\right)$

The curvelet transform can be defined as a function of $\mathrm{x}$ at scale $2^{-\mathrm{j}}$, orientation $\theta_{l}$ and position $\mathrm{x}_{\mathrm{k}}^{(\mathrm{j}, \mathrm{l})}$.

$\varphi_{\mathrm{j}, \mathrm{l}, \mathrm{k}}(\mathrm{x})=\varphi_{\mathrm{j}}\left(\mathrm{R}_{\theta_{\mathrm{l}}}\left(\mathrm{x}-\mathrm{x}_{\mathrm{k}}^{(\mathrm{j}, \mathrm{l})}\right)\right)$

Where $R_{\theta}$ is the rotation in radians. 


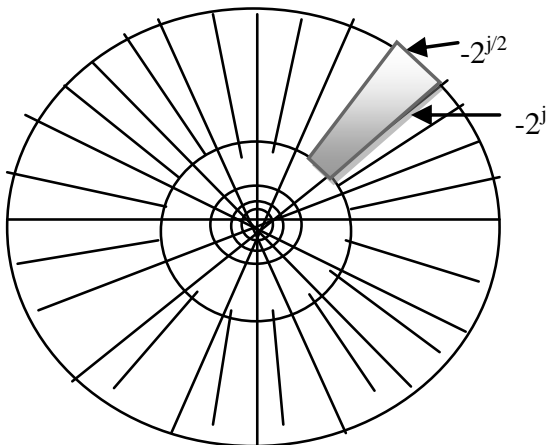

Fig 2: Curvelet tiling of frequency

The elements of curvelet transform obey a special scaling law [7] where the length of the support of frame elements and the width of the support are linked using equation (5).

width $\approx$ length ${ }^{2}$

In this paper we apply discrete curvelet transform on barcode images and compute the statistical features from the transformed images. Curvelet statistical features are then used to represent the barcode images.

\subsection{Neural Network:}

Neural network which is used in computerized processing and that has the advantages of parallel calculating abilities, nonlinear mapping and adaptive ability referred to artificial neural network (ANN).In the field of image processing area various neural network models applied widely. ANN is mostly used to solve the variety of problems of imaging field, like in segmentation, feature extraction, classification and clustering. A neural network is defined as in [8]:

"A neural network is massively parallel and distributed processor made up of simple processing units, which have natural propensity for storing experimental knowledge and making it available for future use". It resembles brain in two aspects:-

1.) Knowledge is acquired by the network through the learning process.

2.) Inter neuron connection strength known as synaptic weight, one used to store this acquired knowledge.

The basic structure of neural network is shown in figure which consists of one input layer, one hidden layer and one output layer.

Input Layer

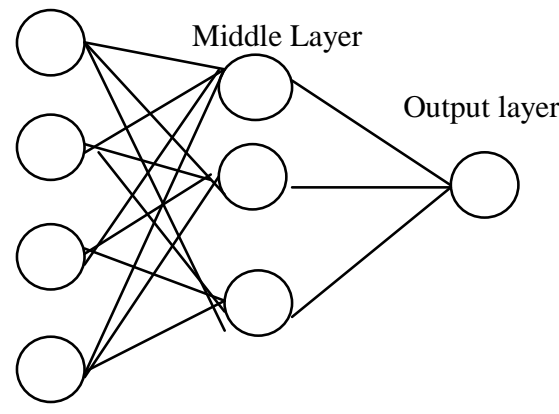

Fig 3: Feed Forward Neural Network
In this paper we use these advantages of neural network for training and testing of barcode images. Process includes creating the network, configuring the network, training and simulating the network etc. Multilayer feed forward network trained with back propagation learning algorithm [9]. This simulated network can be used to locate the barcode in any random image.

\section{METHODOLOGY:}

Neural network training is done by using barcode image database of 50 barcode images. All barcode images are divided into blocks for processing. Then, Curvelet transform is used for computing curvelet feature statistics of each block. After that, Neural Network is trained and tested with these curvelet feature statistics of both positive sample (barcode image) and negative sample (non-barcode image) blocks. Now, this trained neural network can be used to locate the barcode in any image if present. For this following process is performed:

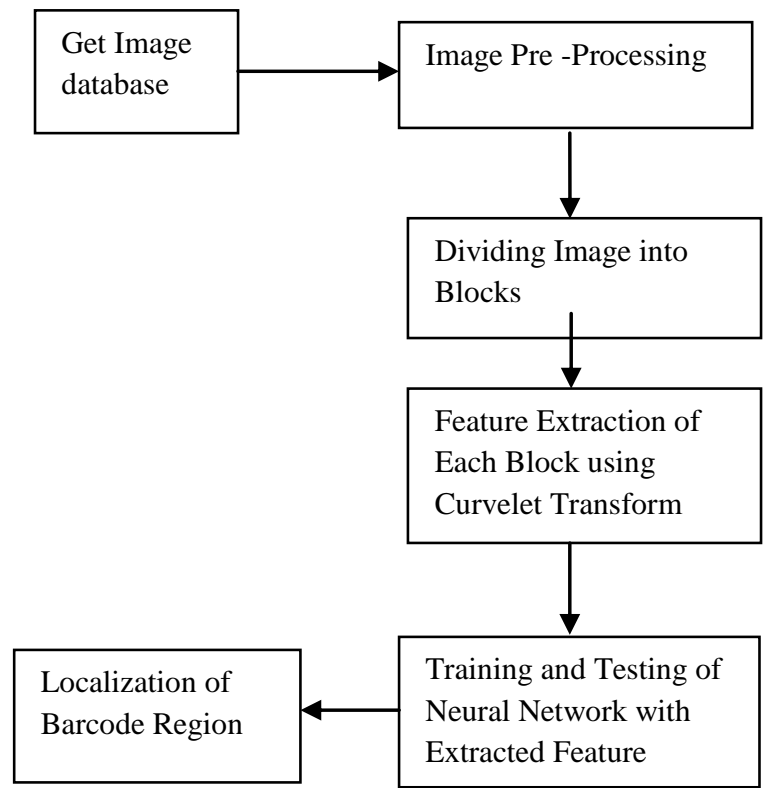

Fig 4: Block Diagram of proposed work.

-The input image (Figure 5)is first resized equal to the size of images used for training of neural network.

-This resized image is divided into non-overlapping blocks of equal size. Figure 6 shows six blocks of blocking process. The block denoted by a, b, c, d captures a part of the barcode, while the block denoted by e, f, g, h captures a part of the texts surrounding the barcode.

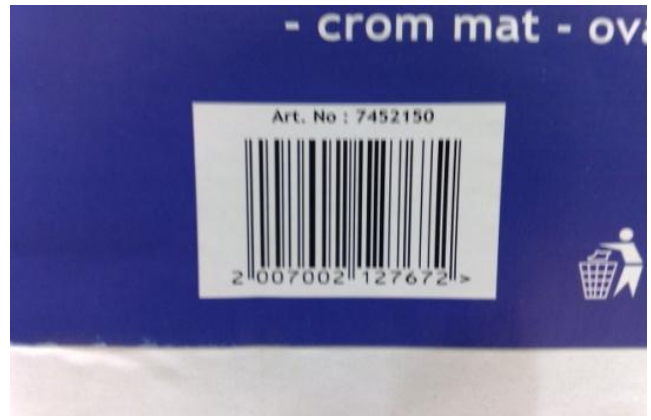

Fig 5: Barcode image captured by a mobile phone. 


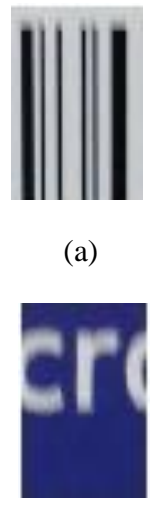

(e)

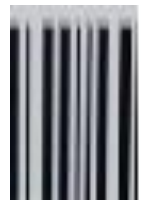

(b)

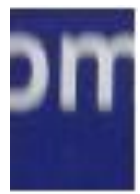

(f)

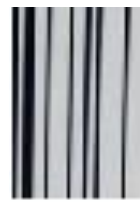

(c)

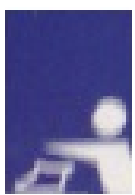

(g)

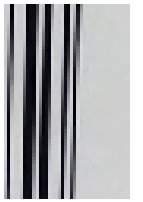

(d)

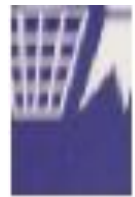

(h)
Fig 6: Some blocks of image shown in fig 5: (a),(b) (c) and (d) are located within barcode region.(e),(f),(g) and (d) blocks are located within other image region.

-For each block, curvelet feature statistics are extracted then mean and standard deviation of these feature are computed by equation (6) and (7), these features are then simulated to the trained neural network.

$$
\begin{aligned}
\mu & =\frac{\sum_{\mathrm{i}=1}^{\mathrm{i}=\mathrm{n}} \mathrm{Xi}}{\mathrm{n}} \\
\sigma & =\sqrt{1 /(n-1) \sum_{i=1}^{i=n}(X i-\mu)^{2}}
\end{aligned}
$$

Here $X_{i}$ is the no of observed value, $n$ is total no observation, $\mu$ is mean and $\sigma$ is std.

-Since, we trained the Neural network with target value 1 for curvelet stastical feature of positive sample i.e. blocks of barcode images and with 0 for curvelet stastical feature of negative samples i.e. blocks of non barcode images.

-This trained neural network will give the value close to target output 1 for image blocks contain barcode portion and for other blokes it will give the value close to target output 0 .

- After that we applied thresholding process to the image blokes, for this 0 is multiplied with block values for which neural network gives the output value less than 0.8 and other blokes will remained as it is.

-At the end output image of thresholding process is converted to logical image by using morphological operation; in this resultant image barcode portion is localized as shown in figure.

\section{RESULTAND PERFORMANCE EVALUTION:}

After training of neural network, some barcode images are tested for performance evaluation of neural network. For example result value obtained by neural network for the image blocks shown in figure (6) of the barcode image shown in figure (5) is given in Table 1:
Table 1: Output value of some block region within image $1 \mathrm{~b}$ by trained Neural Network.

\begin{tabular}{|c|c|}
\hline Block Region Of fig 6 & Output by Neural Network \\
\hline $\mathrm{a}$ & 0.89834 \\
\hline $\mathrm{b}$ & 0.96942 \\
\hline $\mathrm{c}$ & 0.76535 \\
\hline $\mathrm{d}$ & 0.82564 \\
\hline $\mathrm{e}$ & 0.29054 \\
\hline$\hat{\mathrm{g}}$ & -0.04833 \\
\hline$\hat{\mathrm{g}}$ & 0.31241 \\
\hline $\mathrm{h}$ & 0.15246 \\
\hline
\end{tabular}

After obtaining output value for the whole image blocks from trained neural network with curvelet features, the image is converted into binary image .So, the extracted barcode region of image shown in figure(5) is given in figure (7):

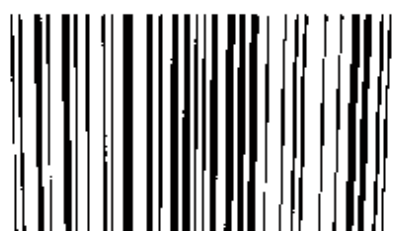

Fig 7: Extracted bar code region of input image Fig1 (b).

The result is also obtained and compared with the technique proposed by Douglas Chai et al.[3].In this technique they detect location of barcode in a image by finding orientation of each labeled region within each block after morphological operation of skeletonizing. This method of barcode localization required that barcode in a image should be in vertical direction so that angles calculated in that block found similar and close to 90.But,in this paper by using curvelet transform which is extracting the features of barcode image blocks. Image blocks containing barcode region have similar feature values as compared to other image blocks. So it will localize the barcode region either barcode present on image captured by mobile phone is in vertical direction or in horizontal direction. For example image shown in fig(8) (a) is captured by mobile phone in which barcode is present the horizontal direction, and the method presented in this paper will efficiently localize this barcode portion shown in fig(8) (b).

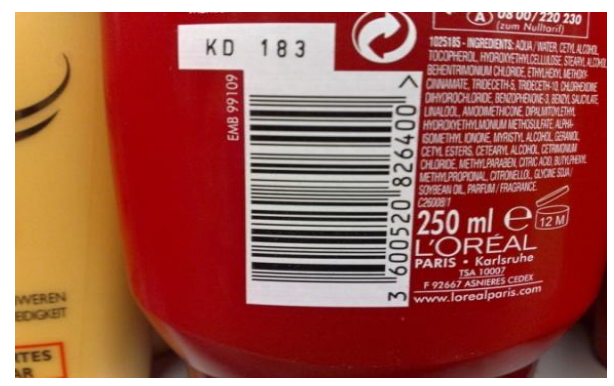

(a) 


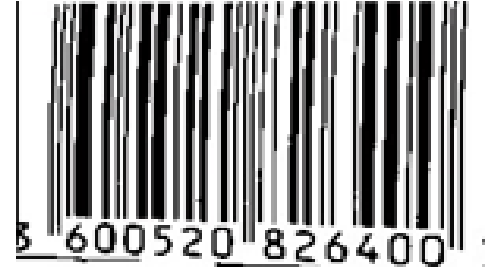

(b)

Fig 8: Barcode image captured by mobile phone with barcode present in horizontal direction(a) and extracted barcode (b) using proposed method.

\section{CONCLUSION:}

In this paper work an image processing based approach is presented to locating EAN-13 barcodes from images captured from smart phones. The proposed methodology extract the features of image using curvelet transform at block level and test these feature values with trained neural network to identify barcode region. This method has a unique advantage that it throws light on the benefits of both curvelet transform and neural network. Since it can detect barcode region present in either vertical or horizontal direction, from mobile captured images. The resultant output is also precise enough for the decoding purpose, so this localized barcode region can further used as precise input for decoding method. The methodology proposed here can also be used to identify other one dimensional barcode images captured by smart phones.

\section{REFERENCES}

[1] 1.Rocholl, J.C., Klenk and Heidemann, "Robust 1D Barcode Recognition on Mobile Devices", in Pattern Recognition (ICPR), 2010 20th International Conference, Istanbul, (OIEEE. doi:10.1109/ICPR.2010.664.

[2] 2. En Peng, Peursum.P. and Ling Li, "Product Barcode and Expiry Date Detection for the Visually Impaired Using a Smartphone", in Digital image computing techniques and applications(DICTA), 2012 international conference, Fremantle, WA, OIEEE, doi : 10.1109/DICTA 2012.6411673.

[3] 3. Chai and Hock, "Locating and Decoding EAN-13 Barcodes from Images Captured by Digital Cameras", in Information, Communications and Signal Processing, 2005 Fifth International Conference, Bangkok, OIEEE, doi:10.1109/ICICS.2005.1689328 .

[4] 4. Katona, Nyul and L.G.,"A Novel Method for Accurate and Efficient Barcode Detection with Morphological Operations", in Signal Image Technology and Internet Based Systems (SITIS), 2012 Eighth International Conference , Naples ,@IEEE, doi 10.1109/SITIS.2012.53

[5] E.J. Candes, D.L. Donoho, Curvelets, multi-resolution representation, and scaling laws, Wavelet Applications in Signal and Image Processing VIII, Vol.4119-01, SPIE, 2000

[6] Guesmi, Tunisia, Trichili, Alimi and Solaiman, "Curvelet transform-based features extraction for fingerprint identification", in Biometrics Special Interest Group (BIOSIG)-Proceedings of the International Conference, 2012, Darmstadt.

[7] AlZubi, Sharif, M.S.,Islam, N. and Abbod, M.,"Multiresolution analysis using curvelet and wavelet transforms for medical imaging", in Medical Measurements and Applications Proceedings (MeMeA), 2011 IEEE International Workshop, 2011,Bari, CIEEE, doi:10.1109/MeMeA.2011.5966687

[8] Haykin Simon, NEURAL NETWORKS: a comprehensive foundation. Prentice Hall PTR .1994.

[9] Svozil, Daniel, Vladimir Kvasnicka, and Jir̂́ Pospichal, "Introduction to multi-layer feed-forward neural networks", in Chemometrics and intelligent laboratory systems 39.1 (1997): 43-62. 